\title{
Article
}

\section{Degree-based topological indices of product graphs}

\author{
Xiaojing Wang ${ }^{1}$, Zhen $\operatorname{Lin}^{2, *}$ and Lianying Miao ${ }^{1}$ \\ 1 School of Mathematics, China University of Mining and Technology, Xuzhou, 221116, Jiangsu, P.R. China. \\ 2 School of Mathematics and Statistics, Qinghai Normal University, Xining, 810008, Qinghai, P.R. China. \\ * Correspondence: lnlinzhen@163.com
}

Academic Editor: Aisha Javed

Received: 21 August 2021; Accepted: 12 October 2021; Published: 1 November 2021.

\begin{abstract}
In this paper, we obtain the quantitative calculation formula of the degree-based topological indices of four standard product for the path and regular graphs, which unify to solve the question on product of these basic graphs without having to deal with it one by one separately. As applications, we give corresponding calculation formula of the general Randić index, the first general Zagreb index and the general sum-connectivity index.
\end{abstract}

Keywords: Degree-based topological indices; Cartesian product; Direct product; Strong product; Lexicographic product.

MSC: 05C07; 05C09; 05C76; 05C90; 05C92.

\section{Introduction}

$\mathbf{T}$ hroughout the article, $G$ is a simple undirected connected graph with vertex set $V(G)$ and edge set $E(G)$. The number of vertices and edges of $G$ is called order and size, respectively. If the vertices $u$ and $v$ are adjacent, then we write $u \sim v$. For $v \in V(G), d_{v}=d_{G}(v)$ denotes the degree of vertex $v$ in $G$. Denote by $P_{n}$ and $K_{1, n-1}$ the path and star with $n$ vertices, respectively.

Cheminformatics is a new interdiscipline composed of chemistry, mathematics and information science, which contributes a major role in the field of chemical sciences by implementing graph theory to mathematical modeling of chemical occurrence. In cheminformatics, the topological indices play a significant role in predicting the biological activities and properties of chemical compounds due to the fact that the numerical characteristics of topological indices reflect certain physico-chemical properties of chemical compounds, such as boiling point, stability, strain energy etc. A large number of topological indices have been studied in the models of Quantitative structure-activity relationships (QSAR) and structure-property relationships (QSPR), such as Wiener index, Randić index, Zagreb index, ABC index and so on.

The study on degree-based topological indices has been one of the hotspots in cheminformatics [1]. Let $K=\{(i, j) \in \mathbb{N} \times \mathbb{N}: 1 \leq i \leq j \leq n-1\}$ and $m_{i, j}=m_{i, j}(G)$ be the number of edges in $G$ joining vertices of degree $i$ and $j$. For any set of numbers $\left\{\varphi_{i, j}\right\}_{(i, j) \in K}$, the general formula of degree-based topological indices is

$$
\operatorname{DTI}(G)=\sum_{(i, j) \in K} m_{i, j}(G) \varphi_{i, j}
$$

In particular, we obtain the first Zagreb index and the second Zagreb index when $\varphi_{i, j}=i+j$ and $\varphi_{i, j}=i j$, respectively.

In 1998, the general Randić index of a graph G, introduced by Bollobás and Erdős [2], is defined as

$$
R^{t}=R^{t}(G)=\sum_{v_{i} v_{j} \in E(G)}\left(d_{i} d_{j}\right)^{t}, \quad t \in \mathbb{R} .
$$

Clearly, we have that $R^{0}$ is the number of edges, $R^{-\frac{1}{2}}$ is the Randić index [3], $R^{-1}$ is the modified second Zagreb index [3], $R^{\frac{1}{2}}$ is the reciprocal Randić index [4], $R^{2}$ is the second Hyper-Zagreb index [4], $R^{1}$ is the second Zagreb index [5], etc. 
In 2005, the first general Zagreb index of a graph $G$ was introduced by Li and Zheng [6] and is defined as

$$
Z^{t}=Z^{t}(G)=\sum_{v_{i} \in V(G)} d_{i}^{t}=\sum_{v_{i} v_{j} \in E(G)}\left(d_{i}^{t-1}+d_{j}^{t-1}\right), \quad t \in \mathbb{R} .
$$

It is easy to see that $Z^{0}$ is the number of vertices, $Z^{1}$ is twice the number of edges, $Z^{2}$ is the first Zagreb index [5], $Z^{3}$ is the forgotten topological index [7], etc.

In 2010, Zhou and Trinajstić [8] proposed the general sum-connectivity index of a graph $G$ as follows:

$$
\chi^{t}=\chi^{t}(G)=\sum_{v_{i} v_{j} \in E(G)}\left(d_{i}+d_{j}\right)^{t}, \quad t \in \mathbb{R} .
$$

It is not difficult to find that $2 \chi^{-1}$ is the harmonic index [9], $\chi^{-\frac{1}{2}}$ is the sum-connectivity index [10], $\chi^{\frac{1}{2}}$ is the reciprocal sum-connectivity index [11], etc.

The product graphs are useful in constructing many important structural models with regularities [12], especially the following four standard product graphs which are widely used in network design [13], multiprocessor system [14], automata theory [15] and other fields. Let $G_{1}$ and $G_{2}$ be two graphs with disjoint vertex sets $\left\{u_{1}, \ldots, u_{m}\right\}$ and $\left\{v_{1}, \ldots, v_{n}\right\}$, respectively. The Cartesian product of $G_{1}$ and $G_{2}$, denoted by $G_{1} \square G_{2}$ is the graph, where $\left(u_{i}, v_{j}\right) \sim\left(u_{r}, v_{s}\right)$ if either $\left(u_{i}=u_{r}\right.$ and $v_{j} \sim v_{s}$ in $\left.G_{2}\right)$ or $\left(u_{i} \sim u_{r}\right.$ in $G_{1}$ and $\left.v_{j}=v_{s}\right)$. The direct product or Kronecker product of $G_{1}$ and $G_{2}$, denoted by $G_{1} \otimes G_{2}$, is the graph where $\left(u_{i}, v_{j}\right) \sim\left(u_{r}, v_{s}\right)$ if $u_{i} \sim u_{r}$ in $G_{1}$ and $v_{j} \sim v_{s}$ in $G_{2}$. The strong product of $G_{1}$ and $G_{2}$, denoted by $G_{1} \otimes G_{2}$, is graph where $\left(u_{i}, u_{j}\right) \sim\left(u_{r}, u_{s}\right)$ if either $\left(u_{i}=u_{r}\right.$ and $u_{j} \sim u_{s}$ in $\left.G_{2}\right)$ or $\left(u_{i} \sim u_{r}\right.$ in $G_{1}$ and $\left.u_{j}=u_{s}\right)$ or $\left(u_{i} \sim u_{r}\right.$ in $G_{1}$ and $u_{j} \sim u_{s}$ in $\left.G_{2}\right)$. The lexicographic product of $G_{1}$ and $G_{2}$, denoted by $G_{1}\left[G_{2}\right]$, is the graph where $\left(u_{i}, v_{j}\right) \sim\left(u_{r}, v_{s}\right)$ if either $\left(u_{i} \sim u_{r}\right.$ in $\left.G_{1}\right)$ or $\left(u_{i}=u_{r}\right.$ and $v_{j} \sim v_{s}$ in $\left.G_{2}\right)$.

In this paper, we give a unified approach to solve the computational problems of degree-based topological indices of standard product graphs for the path and regular graphs, which is generalization of many specific degree-based topological indices. As applications, the corresponding calculation formulas of the general Randić index, the first general Zagreb index and the general sum-connectivity index are obtained.

\section{Cartesian product}

Theorem 1. Let $P_{n_{1}}$ and $P_{n_{2}}$ be two path graphs of order $n_{1}$ and $n_{2}$, respectively. Then

$$
\operatorname{DTI}\left(P_{n_{1}} \square P_{n_{2}}\right)=8 \varphi_{2,3}+2\left(n_{1}+n_{2}-6\right) \varphi_{3,3}+2\left(n_{1}+n_{2}-4\right) \varphi_{3,4}+\left(2 n_{1} n_{2}-5 n_{1}-5 n_{2}+12\right) \varphi_{4,4}
$$

for $n_{1} \geq n_{2} \geq 3$.

Proof. By the definition of Cartesian product, we obtain the basic information on $P_{n_{1}} \square P_{n_{2}}$ in the Table 1 .

Table 1. The basic information on $P_{n_{1}} \square P_{n_{2}}$.

\begin{tabular}{c|c|c|c}
$m_{2,3}$ & $m_{3,3}$ & $m_{3,4}$ & $m_{4,4}$ \\
\hline 8 & $2\left(n_{1}+n_{2}-6\right)$ & $2\left(n_{1}+n_{2}-4\right)$ & $2 n_{1} n_{2}-5 n_{1}-5 n_{2}+12$
\end{tabular}

Thus we have

$\operatorname{DTI}\left(P_{n_{1}} \square P_{n_{2}}\right)=\sum_{(i, j) \in K} m_{i, j}(G) \varphi_{i, j}=8 \varphi_{2,3}+2\left(n_{1}+n_{2}-6\right) \varphi_{3,3}+2\left(n_{1}+n_{2}-4\right) \varphi_{3,4}+\left(2 n_{1} n_{2}-5 n_{1}-5 n_{2}+12\right) \varphi_{4,4} \cdot$

This completes the proof.

Corollary 1. Let $P_{n_{1}}$ and $P_{n_{2}}$ be two path graphs of order $n_{1}$ and $n_{2}$, respectively. Then

$R^{t}\left(P_{n_{1}} \square P_{n_{2}}\right)=8 \cdot 6^{t}+2 \cdot 9^{t}\left(n_{1}+n_{2}-6\right)+2 \cdot 12^{t}\left(n_{1}+n_{2}-4\right)+16^{t}\left(2 n_{1} n_{2}-5 n_{1}-5 n_{2}+12\right) ，$ $Z^{t}\left(P_{n_{1}} \square P_{n_{2}}\right)=8\left(2^{t-1}+3^{t-1}\right)+4 \cdot 3^{t-1}\left(n_{1}+n_{2}-6\right)+2\left(n_{1}+n_{2}-4\right)\left(3^{t-1}+4^{t-1}\right)+2 \cdot 4^{t-1}\left(2 n_{1} n_{2}-5 n_{1}-5 n_{2}+12\right)$, 


$$
\chi^{t}\left(P_{n_{1}} \square P_{n_{2}}\right)=8 \cdot 5^{t}+2 \cdot 6^{t}\left(n_{1}+n_{2}-6\right)+2 \cdot 7^{t}\left(n_{1}+n_{2}-4\right)+8^{t}\left(2 n_{1} n_{2}-5 n_{1}-5 n_{2}+12\right)
$$

for $n_{1} \geq n_{2} \geq 3$.

Theorem 2. Let $P_{n_{1}}$ and $G_{r}$ be a path and a r-regular graph of order $n_{1}$ and $n_{2}$, respectively. Then

$$
\operatorname{DTI}\left(P_{n_{1}} \square G_{r}\right)=r n_{2} \varphi_{r+1, r+1}+2 n_{2} \varphi_{r+1, r+2}+\frac{1}{2}\left[r n_{2}\left(n_{1}-2\right)+2 n_{1} n_{2}-6 n_{2}\right] \varphi_{r+2, r+2}
$$

for $n_{1} \geq n_{2} \geq 2$.

Proof. By the definition of Cartesian product, we obtain the basic information on $P_{n_{1}} \square G_{r}$ in the following Table 2.

Table 2. The basic information on $P_{n_{1}} \square G_{r}$.

\begin{tabular}{c|c|c}
$m_{r+1, r+1}$ & $m_{r+1, r+2}$ & $m_{r+2, r+2}$ \\
\hline$r n_{2}$ & $2 n_{2}$ & $\frac{r n_{2}\left(n_{1}-2\right)}{2}+n_{1} n_{2}-3 n_{2}$
\end{tabular}

Thus we have

$$
\operatorname{DTI}\left(P_{n_{1}} \square G_{r}\right)=\sum_{(i, j) \in K} m_{i, j}(G) \varphi_{i, j}=r n_{2} \varphi_{r+1, r+1}+2 n_{2} \varphi_{r+1, r+2}+\frac{1}{2}\left[r n_{2}\left(n_{1}-2\right)+2 n_{1} n_{2}-6 n_{2}\right] \varphi_{r+2, r+2} .
$$

This completes the proof.

Corollary 2. Let $P_{n_{1}}$ and $G_{r}$ be a path and a r-regular graph of order $n_{1}$ and $n_{2}$, respectively. Then

$$
\begin{aligned}
& R^{t}\left(P_{n_{1}} \square G_{r}\right)=r n_{2}(r+1)^{2 t}+2 n_{2}(r+1)^{t}(r+2)^{t}+\left[\frac{r n_{2}\left(n_{1}-2\right)}{2}+n_{2}\left(n_{1}-3\right)\right](r+2)^{2 t}, \\
& Z^{t}\left(P_{n_{1}} \square G_{r}\right)=2 r n_{2}(r+1)^{t-1}+2 n_{2}\left[(r+1)^{t-1}+(r+2)^{t-1}\right]+2(r+2)^{t-1}\left[\frac{r n_{2}\left(n_{1}-2\right)}{2}+n_{2}\left(n_{1}-3\right)\right], \\
& \chi^{t}\left(P_{n_{1}} \square G_{r}\right)=2^{t} r n_{2}(r+1)^{t}+2 n_{2}(2 r+3)^{t}+2^{t}(r+2)^{t}\left[\frac{r n_{2}\left(n_{1}-2\right)}{2}+n_{2}\left(n_{1}-3\right)\right]
\end{aligned}
$$

for $n_{1} \geq n_{2} \geq 2$.

Theorem 3. Let $G_{r}$ and $P_{n_{2}}$ be a r-regular and a path of order $n_{1}$ and $n_{2}$, respectively. Then

$$
\operatorname{DTI}\left(G_{r} \square P_{n_{2}}\right)=r n_{1} \varphi_{r+1, r+1}+2 n_{1} \varphi_{r+1, r+2}+\frac{1}{2}\left[r n_{1}\left(n_{2}-2\right)+2 n_{1} n_{2}-6 n_{1}\right] \varphi_{r+2, r+2}
$$

for $n_{1} \geq n_{2} \geq 2$.

Proof. By the definition of Cartesian product, we obtain the basic information on $G_{r} \square P_{n_{2}}$ in the following Table 3.

Table 3. The basic information on $G_{r} \square P_{n_{2}}$.

\begin{tabular}{c|c|c}
$m_{r+1, r+1}$ & $m_{r+1, r+2}$ & $m_{r+2, r+2}$ \\
\hline$r n_{1}$ & $2 n_{1}$ & $\frac{r n_{1}\left(n_{2}-2\right)}{2}+n_{1} n_{2}-3 n_{1}$
\end{tabular}

Thus we have

$$
\operatorname{DTI}\left(G_{r} \square P_{n_{2}}\right)=\sum_{(i, j) \in K} m_{i, j}(G) \varphi_{i, j}=r n_{1} \varphi_{r+1, r+1}+2 n_{1} \varphi_{r+1, r+2}+\frac{1}{2}\left[r n_{1}\left(n_{2}-2\right)+2 n_{1} n_{2}-6 n_{1}\right] \varphi_{r+2, r+2 .}
$$


This completes the proof.

Corollary 3. Let $G_{r}$ and $P_{n_{2}}$ be a r-regular and a path of order $n_{1}$ and $n_{2}$, respectively. Then

$$
\begin{aligned}
& R^{t}\left(G_{r} \square P_{n_{2}}\right)=r n_{1}(r+1)^{2 t}+2 n_{1}(r+1)^{t}(r+2)^{t}+\left[\frac{r n_{1}\left(n_{2}-2\right)}{2}+n_{1}\left(n_{2}-3\right)\right](r+2)^{2 t}, \\
& Z^{t}\left(G_{r} \square P_{n_{2}}\right)=2 r n_{1}(r+1)^{t-1}+2 n_{1}\left[(r+1)^{t-1}+(r+2)^{t-1}\right]+2(r+2)^{t-1}\left[\frac{r n_{1}\left(n_{2}-2\right)}{2}+n_{1}\left(n_{2}-3\right)\right], \\
& \chi^{t}\left(G_{r} \square P_{n_{2}}\right)=2^{t} r n_{1}(r+1)^{t}+2 n_{1}(2 r+3)^{t}+2^{t}(r+2)^{t}\left[\frac{r n_{1}\left(n_{2}-2\right)}{2}+n_{1}\left(n_{2}-3\right)\right]
\end{aligned}
$$

for $n_{1} \geq n_{2} \geq 2$.

Theorem 4. Let $G_{1}$ and $G_{2}$ be a $r_{1}$-regular graph and a $r_{2}$-regular graph with order $n_{1}$ and $n_{2}$, respectively. Then

$$
\operatorname{DTI}\left(G_{1} \square G_{2}\right)=\frac{n_{1} n_{2}\left(r_{1}+r_{2}\right)}{2} \varphi_{r_{1}+r_{2}, r_{1}+r_{2}}
$$

for $n_{1} \geq n_{2} \geq 2$.

Proof. By the definition of Cartesian product, we have $G_{1} \square G_{2}$ is a $\left(r_{1}+r_{2}\right)$-regular graph with $\frac{n_{1} n_{2}\left(r_{1}+r_{2}\right)}{2}$ edges. Thus

$$
\operatorname{DTI}\left(G_{1} \square G_{2}\right)=\sum_{(i, j) \in K} m_{i, j}(G) \varphi_{i, j}=\frac{n_{1} n_{2}\left(r_{1}+r_{2}\right)}{2} \varphi_{r_{1}+r_{2}, r_{1}+r_{2}} .
$$

This completes the proof.

Corollary 4. Let $G_{1}$ and $G_{2}$ be a $r_{1}$-regular graph and a $r_{2}$-regular graph with order $n_{1}$ and $n_{2}$, respectively. Then

$$
\begin{aligned}
& R^{t}\left(G_{1} \square G_{2}\right)=\frac{n_{1} n_{2}\left(r_{1}+r_{2}\right)^{2 t+1}}{2}, \\
& Z^{t}\left(G_{1} \square G_{2}\right)=n_{1} n_{2}\left(r_{1}+r_{2}\right)^{t}, \\
& \chi^{t}\left(G_{1} \square G_{2}\right)=2^{t-1} n_{1} n_{2}\left(r_{1}+r_{2}\right)^{t+1}
\end{aligned}
$$

for $n_{1} \geq n_{2} \geq 2$.

\section{Direct product}

Theorem 5. Let $P_{n_{1}}$ and $P_{n_{2}}$ be two path graphs of order $n_{1}$ and $n_{2}$, respectively. Then

$$
\operatorname{DTI}\left(P_{n_{1}} \otimes P_{n_{2}}\right)=4 \varphi_{1,4}+4 \varphi_{2,2}+4\left(n_{1}+n_{2}-6\right) \varphi_{2,4}+2\left(n_{1}-3\right)\left(n_{2}-3\right) \varphi_{4,4}
$$

for $n_{1} \geq n_{2} \geq 3$.

Proof. By the definition of direct product, we obtain the basic information on $P_{n_{1}} \otimes P_{n_{2}}$ in the following Table 4.

Table 4. The basic information on $P_{n_{1}} \otimes P_{n_{2}}$.

\begin{tabular}{c|c|c|c}
$m_{1,4}$ & $m_{2,2}$ & $m_{2,4}$ & $m_{4,4}$ \\
\hline 4 & 4 & $4\left(n_{1}+n_{2}-6\right)$ & $2\left(n_{1}-3\right)\left(n_{2}-3\right)$
\end{tabular}

Thus we have

$$
\operatorname{DTI}\left(P_{n_{1}} \otimes P_{n_{2}}\right)=\sum_{(i, j) \in K} m_{i, j}(G) \varphi_{i, j}=4 \varphi_{1,4}+4 \varphi_{2,2}+4\left(n_{1}+n_{2}-6\right) \varphi_{2,4}+2\left(n_{1}-3\right)\left(n_{2}-3\right) \varphi_{4,4} .
$$


This completes the proof.

Corollary 5. Let $P_{n_{1}}$ and $P_{n_{2}}$ be two path graphs of order $n_{1}$ and $n_{2}$, respectively. Then

$$
\begin{aligned}
& R^{t}\left(P_{n_{1}} \otimes P_{n_{2}}\right)=8 \cdot 4^{t}+2 \cdot 8^{t}\left(n_{1} n_{2}-n_{1}-n_{2}-3\right), \\
& Z^{t}\left(P_{n_{1}} \otimes P_{n_{2}}\right)=4\left[1+2^{t}+\left(n_{1}+n_{2}-6\right)\left(2^{t-1}+4^{t-1}\right)\right]+4^{t}\left[1+\left(n_{1}-3\right)\left(n_{2}-3\right)\right], \\
& \chi^{t}\left(P_{n_{1}} \otimes P_{n_{2}}\right)=4\left[4^{t}+5^{t}+6^{t}\left(n_{1}+n_{2}-6\right)\right]+2^{3 t+1}\left(n_{1}-3\right)\left(n_{2}-3\right)
\end{aligned}
$$

for $n_{1} \geq n_{2} \geq 3$.

Theorem 6. Let $P_{n_{1}}$ and $G_{r}$ be a path and a r-regular of order $n_{1}$ and $n_{2}$, respectively. Then

$$
\operatorname{DTI}\left(P_{n_{1}} \otimes G_{r}\right)=2 r n_{2} \varphi_{r, 2 r}+r n_{2}\left(n_{1}-3\right) \varphi_{2 r, 2 r}
$$

for $n_{1} \geq n_{2} \geq 3$.

Proof. By the definition of direct product, we obtain the basic information on $P_{n_{1}} \otimes G_{r}$ in the following Table 5.

Table 5. The basic information on $P_{n_{1}} \otimes G_{r}$.

$$
\begin{array}{c|c}
m_{r, 2 r} & m_{2 r, 2 r} \\
\hline 2 r n_{2} & r n_{2}\left(n_{1}-3\right)
\end{array}
$$

Thus we have

$$
\operatorname{DTI}\left(P_{n_{1}} \otimes G_{r}\right)=\sum_{(i, j) \in K} m_{i, j}(G) \varphi_{i, j}=2 r n_{2} \varphi_{r, 2 r}+r n_{2}\left(n_{1}-3\right) \varphi_{2 r, 2 r} .
$$

This completes the proof.

Corollary 6. Let $P_{n_{1}}$ and $G_{r}$ be a path and a r-regular of order $n_{1}$ and $n_{2}$, respectively. Then

$$
\begin{aligned}
& R^{t}\left(P_{n_{1}} \otimes G_{r}\right)=2^{t+1} r^{2 t+1} n_{2}+2^{2 t} r^{2 t+1} n_{2}\left(n_{1}-3\right), \\
& Z^{t}\left(P_{n_{1}} \otimes G_{r}\right)=\left(2+2^{t}\right) r^{t} n_{2}+2^{t} r^{t} n_{2}\left(n_{1}-3\right), \\
& \chi^{t}\left(P_{n_{1}} \otimes G_{r}\right)=2 n_{2} \cdot 3^{t} \cdot r^{t+1}+4^{t} r^{t+1} n_{2}\left(n_{1}-3\right)
\end{aligned}
$$

for $n_{1} \geq n_{2} \geq 3$.

Theorem 7. Let $G_{r}$ and $P_{n_{2}}$ be a r-regular and a path of order $n_{1}$ and $n_{2}$, respectively. Then

$$
\operatorname{DTI}\left(G_{r} \otimes P_{n_{2}}\right)=2 r n_{1} \varphi_{r, 2 r}+r n_{1}\left(n_{2}-3\right) \varphi_{2 r, 2 r}
$$

for $n_{1} \geq n_{2} \geq 3$.

Proof. By the definition of direct product, we obtain the basic information on $G_{r} \otimes P_{n_{2}}$ in the following Table 6.

Table 6. The basic information on $G_{r} \otimes P_{n_{2}}$.

$$
\begin{array}{c|c}
m_{r, 2 r} & m_{2 r, 2 r} \\
\hline 2 r n_{1} & r n_{1}\left(n_{2}-3\right)
\end{array}
$$

Thus we have

$$
\operatorname{DTI}\left(G_{r} \otimes P_{n_{2}}\right)=\sum_{(i, j) \in K} m_{i, j}(G) \varphi_{i, j}=2 r n_{1} \varphi_{r, 2 r}+r n_{1}\left(n_{2}-3\right) \varphi_{2 r, 2 r} .
$$

This completes the proof. 
Corollary 7. Let $G_{r}$ and $P_{n_{2}}$ be a r-regular and a path of order $n_{1}$ and $n_{2}$, respectively. Then

$$
\begin{aligned}
& R^{t}\left(G_{r} \otimes P_{n_{2}}\right)=2^{t+1} r^{2 t+1} n_{1}+2^{2 t} r^{2 t+1} n_{1}\left(n_{2}-3\right), \\
& Z^{t}\left(G_{r} \otimes P_{n_{2}}\right)=r^{t} n_{1}\left(2+2^{t}\right)+2^{t} r^{t} n_{1}\left(n_{2}-3\right), \\
& \chi^{t}\left(G_{r} \otimes P_{n_{2}}\right)=2 \cdot 3^{t} \cdot r^{t+1} n_{1}+4^{t} r^{t+1} n_{1}\left(n_{2}-3\right)
\end{aligned}
$$

for $n_{1} \geq n_{2} \geq 3$.

Theorem 8. Let $G_{1}$ and $G_{2}$ be a $r_{1}$-regular graph and a $r_{2}$-regular graph with order $n_{1}$ and $n_{2}$, respectively. Then

$$
\operatorname{DTI}\left(G_{1} \otimes G_{2}\right)=\frac{r_{1} r_{2} n_{1} n_{2}}{2} \varphi_{r_{1} r_{2}, r_{1} r_{2}}
$$

for $n_{1} \geq n_{2} \geq 2$.

Proof. By the definition of direct product, we have $G_{1} \otimes G_{2}$ is a $r_{1} r_{2}$-regular graph with $\frac{r_{1} r_{2} n_{1} n_{2}}{2}$ edges. Thus

$$
\operatorname{DTI}\left(G_{1} \otimes G_{2}\right)=\sum_{(i, j) \in K} m_{i, j}(G) \varphi_{i, j}=\frac{r_{1} r_{2} n_{1} n_{2}}{2} \varphi_{r_{1} r_{2}, r_{1} r_{2}} .
$$

This completes the proof.

Corollary 8. Let $G_{1}$ and $G_{2}$ be a $r_{1}$-regular graph and a $r_{2}$-regular graph with order $n_{1}$ and $n_{2}$, respectively. Then

$$
\begin{aligned}
& R^{t}\left(G_{1} \otimes G_{2}\right)=\frac{n_{1} n_{2}\left(r_{1} r_{2}\right)^{2 t+1}}{2} \\
& Z^{t}\left(G_{1} \otimes G_{2}\right)=n_{1} n_{2}\left(r_{1} r_{2}\right)^{t} \\
& \chi^{t}\left(G_{1} \otimes G_{2}\right)=2^{t-1} n_{1} n_{2}\left(r_{1} r_{2}\right)^{t+1}
\end{aligned}
$$

for $n_{1} \geq n_{2} \geq 2$.

\section{Strong product}

Theorem 9. Let $P_{n_{1}}$ and $P_{n_{2}}$ be two path graphs of order $n_{1}$ and $n_{2}$, respectively. Then

$$
\operatorname{DTI}\left(P_{n_{1}} \otimes P_{n_{2}}\right)=8 \varphi_{3,5}+4 \varphi_{3,8}+2\left(n_{1}+n_{2}-4\right) \varphi_{5,5}+\left(6 n_{1}+6 n_{2}-32\right) \varphi_{5,8}+\left[4 n_{1} n_{2}-11\left(n_{1}+n_{2}\right)+30\right] \varphi_{8,8}
$$

for $n_{1} \geq n_{2} \geq 3$.

Proof. By the definition of strong product, we obtain the basic information on $P_{n_{1}} \otimes P_{n_{2}}$ in the following Table 7.

Table 7. The basic information on $P_{n_{1}} \otimes P_{n_{2}}$.

\begin{tabular}{c|c|c|c|c}
$m_{3,5}$ & $m_{3,8}$ & $m_{5,5}$ & $m_{5,8}$ & $m_{8,8}$ \\
\hline 8 & 4 & $2\left(n_{1}+n_{2}\right)-8$ & $6\left(n_{1}+n_{2}\right)-32$ & $4 n_{1} n_{2}-11\left(n_{1}+n_{2}\right)+30$
\end{tabular}

Thus we have

$$
\begin{aligned}
\operatorname{DTI}\left(P_{n_{1}} \otimes P_{n_{2}}\right) & =\sum_{(i, j) \in K} m_{i, j}(G) \varphi_{i, j} \\
& =8 \varphi_{3,5}+4 \varphi_{3,8}+2\left(n_{1}+n_{2}-4\right) \varphi_{5,5}+\left(6 n_{1}+6 n_{2}-32\right) \varphi_{5,8}+\left[4 n_{1} n_{2}-11\left(n_{1}+n_{2}\right)+30\right] \varphi_{8,8} .
\end{aligned}
$$

This completes the proof. 
Corollary 9. Let $P_{n_{1}}$ and $P_{n_{2}}$ be two path graphs of order $n_{1}$ and $n_{2}$, respectively. Then

$$
\begin{aligned}
R^{t}\left(P_{n_{1}} \otimes P_{n_{2}}\right)= & 8 \cdot 15^{t}+4 \cdot 24^{t}+25^{t} \cdot\left[2\left(n_{1}+n_{2}-4\right)\right]+40^{t} \cdot\left(6 n_{1}+6 n_{2}-32\right) \\
& +64^{t} \cdot\left[4 n_{1} n_{2}-11\left(n_{1}+n_{2}\right)+30\right], \\
Z^{t}\left(P_{n_{1}} \otimes P_{n_{2}}\right)= & 8 \cdot\left(3^{t-1}+5^{t-1}\right)+4 \cdot\left(3^{t-1}+8^{t-1}\right)+4 \cdot 5^{t-1}\left[\left(n_{1}+n_{2}\right)-4\right] \\
& +\left(6 n_{1}+6 n_{2}-32\right) \cdot\left(5^{t-1}+8^{t-1}\right)+2 \cdot 8^{t-1}\left[4 n_{1} n_{2}-11\left(n_{1}+n_{2}\right)+30\right], \\
\chi^{t}\left(P_{n_{1}} \otimes P_{n_{2}}\right)= & 8^{t+1}+4 \cdot 11^{t}+10^{t} \cdot\left[2\left(n_{1}+n_{2}\right)-8\right]+13^{t} \cdot\left(6 n_{1}+6 n_{2}-32\right) \\
& +16^{t} \cdot\left[4 n_{1} n_{2}-11\left(n_{1}+n_{2}\right)+30\right]
\end{aligned}
$$

for $n_{1} \geq n_{2} \geq 3$.

Theorem 10. Let $P_{n_{1}}$ and $G_{r}$ be a path and a r-regular of order $n_{1}$ and $n_{2}$, respectively. Then

$$
\operatorname{DTI}\left(P_{n_{1}} \otimes G_{r}\right)=r n_{2} \varphi_{2 r+1,2 r+1}+2(r+1) n_{2} \varphi_{2 r+1,3 r+2}+\frac{1}{2}\left[n_{1} n_{2}(3 r+2)-2 n_{2}(4 r+3)\right] \varphi_{3 r+2,3 r+2}
$$

for $n_{1}>n_{2} \geq 2$.

Proof. By the definition of strong product, we obtain the basic information on $P_{n_{1}} \otimes G_{r}$ in the following Table 8.

Table 8. The basic information on $P_{n_{1}} \otimes G_{r}$.

\begin{tabular}{c|c|c}
$m_{2 r+1,2 r+1}$ & $m_{2 r+1,3 r+2}$ & $m_{3 r+2,3 r+2}$ \\
\hline$r n_{2}$ & $2(r+1) n_{2}$ & $n_{1} n_{2}\left(\frac{3 r}{2}+1\right)-n_{2}(4 r+3)$
\end{tabular}

Thus we have

$$
\begin{aligned}
\operatorname{DTI}\left(P_{n_{1}} \otimes G_{r}\right) & =\sum_{(i, j) \in K} m_{i, j}(G) \varphi_{i, j} \\
& =r n_{2} \varphi_{2 r+1,2 r+1}+2(r+1) n_{2} \varphi_{2 r+1,3 r+2}+\frac{1}{2}\left[n_{1} n_{2}(3 r+2)-2 n_{2}(4 r+3)\right] \varphi_{3 r+2,3 r+2} .
\end{aligned}
$$

This completes the proof.

Corollary 10. Let $P_{n_{1}}$ and $G_{r}$ be a path and a r-regular of order $n_{1}$ and $n_{2}$, respectively. Then

$$
\begin{aligned}
& R^{t}\left(P_{n_{1}} \otimes G_{r}\right)=r n_{2}(2 r+1)^{2 t}+2 n_{2}(r+1)(2 r+1)^{t}(3 r+2)^{t}+\left[n_{1} n_{2}\left(\frac{3 r}{2}+1\right)-n_{2}(4 r+3)\right](3 r+2)^{2 t}, \\
& Z^{t}\left(P_{n_{1}} \otimes G_{r}\right)=2 r n_{2}(2 r+1)^{t-1}+2 n_{2}(r+1)\left[(2 r+1)^{t-1}+(3 r+2)^{t-1}\right]+2(3 r+2)^{t-1}\left[n_{1} n_{2}\left(\frac{3 r}{2}+1\right)-n_{2}(4 r+3)\right] \\
& \chi^{t}\left(P_{n_{1}} \otimes G_{r}\right)=2^{t} r n_{2}(2 r+1)^{t}+2 n_{2}(r+1)(5 r+3)^{t}+2^{t}(3 r+2)^{t}\left[n_{1} n_{2}\left(\frac{3 r}{2}+1\right)-n_{2}(4 r+3)\right]
\end{aligned}
$$

for $n_{1}>n_{2} \geq 2$.

Theorem 11. Let $G_{r}$ and $P_{n_{2}}$ be a r-regular and a path of order $n_{1}$ and $n_{2}$, respectively. Then

$$
\operatorname{DTI}\left(G_{r} \otimes P_{n_{2}}\right)=r n_{1} \varphi_{2 r+1,2 r+1}+2(r+1) n_{1} \varphi_{2 r+1,3 r+2}+\frac{1}{2}\left[n_{1} n_{2}(3 r+2)-2 n_{1}(4 r+3)\right] \varphi_{3 r+2,3 r+2}
$$

for $n_{1} \geq n_{2} \geq 3$.

Proof. By the definition of strong product, we obtain the basic information on $G_{r} \otimes P_{n_{2}}$ in the following Table 9. 
Table 9. The basic information on $G_{r} \otimes P_{n_{2}}$.

\begin{tabular}{c|c|c}
$m_{2 r+1,2 r+1}$ & $m_{2 r+1,3 r+2}$ & $m_{3 r+2,3 r+2}$ \\
\hline$r n_{1}$ & $2(r+1) n_{1}$ & $n_{1} n_{2}\left(\frac{3 r}{2}+1\right)-n_{1}(4 r+3)$
\end{tabular}

Thus we have

$$
\begin{aligned}
\operatorname{DTI}\left(G_{r} \otimes P_{n_{2}}\right) & =\sum_{(i, j) \in K} m_{i, j}(G) \varphi_{i, j} \\
& =r n_{1} \varphi_{2 r+1,2 r+1}+2(r+1) n_{1} \varphi_{2 r+1,3 r+2}+\frac{1}{2}\left[n_{1} n_{2}(3 r+2)-2 n_{1}(4 r+3)\right] \varphi_{3 r+2,3 r+2 .}
\end{aligned}
$$

This completes the proof.

Corollary 11. Let $G_{r}$ and $P_{n_{2}}$ be a r-regular and a path of order $n_{1}$ and $n_{2}$, respectively. Then

$$
\begin{aligned}
& R^{t}\left(G_{r} \otimes P_{n_{2}}\right)=r n_{1}(2 r+1)^{2 t}+2 n_{1}(r+1)(2 r+1)^{t}(3 r+2)^{t}+(3 r+2)^{2 t}\left[n_{1} n_{2}\left(\frac{3 r}{2}+1\right)-n_{1}(4 r+3)\right], \\
& Z^{t}\left(G_{r} \otimes P_{n_{2}}\right)=2 r n_{1}(2 r+1)^{t-1}+2 n_{1}(r+1)\left[(2 r+1)^{t-1}+(3 r+2)^{t-1}\right]+2(3 r+2)^{t-1}\left[n_{1} n_{2}\left(\frac{3 r}{2}+1\right)-n_{1}(4 r+3)\right], \\
& \chi^{t}\left(G_{r} \otimes P_{n_{2}}\right)=2^{t} r n_{1}(2 r+1)^{t}+2 n_{1}(r+1)(5 r+3)^{t}+2^{t}(3 r+2)^{t}\left[n_{1} n_{2}\left(\frac{3 r}{2}+1\right)-n_{1}(4 r+3)\right]
\end{aligned}
$$

for $n_{1} \geq n_{2} \geq 3$.

Theorem 12. Let $G_{1}$ and $G_{2}$ be a $r_{1}$-regular graph and a $r_{2}$-regular graph with order $n_{1}$ and $n_{2}$, respectively. Then

$$
\operatorname{DTI}\left(G_{1} \otimes G_{2}\right)=\frac{n_{1} n_{2}\left(r_{1} r_{2}+r_{1}+r_{2}\right)}{2} \varphi_{r_{1} r_{2}+r_{1}+r_{2}, r_{1} r_{2}+r_{1}+r_{2}}
$$

for $n_{1} \geq n_{2} \geq 2$.

Proof. By the definition of strong product, we have $G_{1} \otimes G_{2}$ is a $\left(r_{1} r_{2}+r_{1}+r_{2}\right)$-regular graph with $\frac{n_{1} n_{2}\left(r_{1} r_{2}+r_{1}+r_{2}\right)}{2}$ edges. Thus

$$
\operatorname{DTI}\left(G_{1} \otimes G_{2}\right)=\sum_{(i, j) \in K} m_{i, j}(G) \varphi_{i, j}=\frac{n_{1} n_{2}\left(r_{1} r_{2}+r_{1}+r_{2}\right)}{2} \varphi_{r_{1} r_{2}+r_{1}+r_{2}, r_{1} r_{2}+r_{1}+r_{2}} .
$$

This completes the proof.

Corollary 12. Let $G_{1}$ and $G_{2}$ be a $r_{1}$-regular graph and a $r_{2}$-regular graph with order $n_{1}$ and $n_{2}$, respectively. Then

$$
\begin{aligned}
& R^{t}\left(G_{1} \otimes G_{2}\right)=\frac{n_{1} n_{2}\left(r_{1} r_{2}+r_{1}+r_{2}\right)^{2 t+1}}{2}, \\
& Z^{t}\left(G_{1} \otimes G_{2}\right)=n_{1} n_{2}\left(r_{1} r_{2}+r_{1}+r_{2}\right)^{t}, \\
& \chi^{t}\left(G_{1} \otimes G_{2}\right)=2^{t-1} n_{1} n_{2}\left(r_{1} r_{2}+r_{1}+r_{2}\right)^{t+1}
\end{aligned}
$$

for $n_{1} \geq n_{2} \geq 2$.

\section{Lexicographic product}

Theorem 13. Let $P_{n_{1}}$ and $P_{n_{2}}$ be two path graphs of order $n_{1}$ and $n_{2}$, respectively. Then

$$
\begin{aligned}
\operatorname{DTI}\left(P_{n_{1}}\left[P_{n_{2}}\right]\right) & =4 \varphi_{n_{2}+1, n_{2}+2}+8 \varphi_{n_{2}+1,2 n_{2}+1}+4\left(n_{2}-2\right) \varphi_{n_{2}+1,2 n_{2}+2}+2\left(n_{2}-3\right) \varphi_{n_{2}+2, n_{2}+2}+4\left(n_{2}-2\right) \varphi_{n_{2}+2,2 n_{2}+1} \\
& +2\left(n_{2}-2\right)^{2} \varphi_{n_{2}+2,2 n_{2}+2}+4\left(n_{1}-3\right) \varphi_{2 n_{2}+1,2 n_{2}+1}+\left[2\left(n_{1}-2\right)+4\left(n_{1}-3\right)\left(n_{2}-2\right)\right] \varphi_{2 n_{2}+1,2 n_{2}+2} \\
& +\left[\left(n_{1}-2\right)\left(n_{2}-3\right)+\left(n_{1}-3\right)\left(n_{2}-2\right)^{2}\right] \varphi_{2 n_{2}+2,2 n_{2}+2}
\end{aligned}
$$


for $n_{1} \geq n_{2} \geq 3$.

Proof. By the definition of lexicographic product, we obtain the basic information on $P_{n_{1}}\left[P_{n_{2}}\right]$ in the following Table 10.

Table 10. The basic information on $P_{n_{1}}\left[P_{n_{2}}\right]$.

\begin{tabular}{c|c}
$m_{n_{2}+1, n_{2}+2}$ & 4 \\
\hline$m_{n_{2}+1,2 n_{2}+1}$ & 8 \\
\hline$m_{n_{2}+1,2 n_{2}+2}$ & $4\left(n_{2}-2\right)$ \\
\hline$m_{n_{2}+2, n_{2}+2}$ & $2\left(n_{2}-3\right)$ \\
\hline$m_{n_{2}+2,2 n_{2}+1}$ & $4\left(n_{2}-2\right)$ \\
\hline$m_{n_{2}+2,2 n_{2}+2}$ & $2\left(n_{2}-2\right)^{2}$ \\
\hline$m_{2 n_{2}+1,2 n_{2}+1}$ & $4\left(n_{1}-3\right)$ \\
\hline$m_{2 n_{2}+1,2 n_{2}+2}$ & $2\left(n_{1}-2\right)+4\left(n_{1}-3\right)\left(n_{2}-2\right)$ \\
\hline$m_{2 n_{2}+2,2 n_{2}+2}$ & $\left(n_{1}-2\right)\left(n_{2}-3\right)+\left(n_{1}-3\right)\left(n_{2}-2\right)^{2}$
\end{tabular}

Thus we have

$$
\begin{aligned}
\operatorname{DTI}\left(P_{n_{1}}\left[P_{n_{2}}\right]\right)= & \sum_{(i, j) \in K} m_{i, j}(G) \varphi_{i, j} \\
= & 4 \varphi_{n_{2}+1, n_{2}+2}+8 \varphi_{n_{2}+1,2 n_{2}+1}+4\left(n_{2}-2\right) \varphi_{n_{2}+1,2 n_{2}+2} \\
& +2\left(n_{2}-3\right) \varphi_{n_{2}+2, n_{2}+2}+4\left(n_{2}-2\right) \varphi_{n_{2}+2,2 n_{2}+1}+2\left(n_{2}-2\right)^{2} \varphi_{n_{2}+2,2 n_{2}+2} \\
& +4\left(n_{1}-3\right) \varphi_{2 n_{2}+1,2 n_{2}+1}+\left[2\left(n_{1}-2\right)+4\left(n_{1}-3\right)\left(n_{2}-2\right)\right] \varphi_{2 n_{2}+1,2 n_{2}+2} \\
& +\left[\left(n_{1}-2\right)\left(n_{2}-3\right)+\left(n_{1}-3\right)\left(n_{2}-2\right)^{2}\right] \varphi_{2 n_{2}+2,2 n_{2}+2 .}
\end{aligned}
$$

This completes the proof.

Corollary 13. Let $P_{n_{1}}$ and $P_{n_{2}}$ be two path graphs of order $n_{1}$ and $n_{2}$, respectively. Then

$$
\begin{aligned}
R^{t}\left(P_{n_{1}}\left[P_{n_{2}}\right]\right)= & 4\left[\left(n_{2}+1\right)\left(n_{2}+2\right)\right]^{t}+8\left[\left(n_{2}+1\right)\left(2 n_{2}+1\right)\right]^{t}+4\left(n_{2}-2\right)\left[\left(n_{2}+1\right)\left(2 n_{2}+2\right)\right]^{t} \\
& +2\left(n_{2}-3\right)\left(n_{2}+2\right)^{2 t}+4\left(n_{2}-2\right)\left[\left(n_{2}+2\right)\left(2 n_{2}+1\right)\right]^{t} \\
& +2\left(n_{2}-2\right)^{2}\left[\left(n_{2}+2\right)\left(2 n_{2}+2\right)\right]^{t}+4\left(n_{1}-3\right)\left(2 n_{2}+1\right)^{2 t} \\
& +\left[2\left(n_{1}-2\right)+4\left(n_{1}-3\right)\left(n_{2}-2\right)\right]\left[\left(2 n_{2}+1\right)\left(2 n_{2}+2\right)\right]^{t} \\
& +\left[\left(n_{1}-2\right)\left(n_{2}-3\right)+\left(n_{1}-3\right)\left(n_{2}-2\right)^{2}\right]\left(2 n_{2}+2\right)^{2 t}, \\
Z^{t}\left(P_{n_{1}}\left[P_{n_{2}}\right]\right)= & 4\left[\left(n_{2}+1\right)^{t-1}+\left(n_{2}+2\right)^{t-1}\right]+8\left[\left(n_{2}+1\right)^{t-1}+\left(2 n_{2}+1\right)^{t-1}\right] \\
& +4\left(n_{2}-2\right)\left[\left(n_{2}+1\right)^{t-1}+\left(2 n_{2}+2\right)^{t-1}\right]+4\left(n_{2}-3\right)\left(n_{2}+2\right)^{t-1} \\
& +4\left(n_{2}-2\right)\left[\left(n_{2}+2\right)^{t-1}+\left(2 n_{2}+1\right)^{t-1}\right]+2\left(n_{2}-2\right)^{2}\left[\left(n_{2}+2\right)^{t-1}\right. \\
& \left.+\left(2 n_{2}+2\right)^{t-1}\right]+8\left(n_{1}-3\right)\left(2 n_{2}+1\right)^{t-1} \\
& +\left[2\left(n_{1}-2\right)+4\left(n_{1}-3\right)\left(n_{2}-2\right)\right]\left[\left(2 n_{2}+1\right)^{t-1}+\left(2 n_{2}+2\right)^{t-1}\right] \\
& +2\left(2 n_{2}+2\right)^{t-1}\left[\left(n_{1}-2\right)\left(n_{2}-3\right)+\left(n_{1}-3\right)\left(n_{2}-2\right)^{2}\right], \\
\chi^{t}\left(P_{n_{1}}\left[P_{n_{2}}\right]\right)= & 4\left(2 n_{2}+3\right)^{t}+8\left(3 n_{2}+2\right)^{t}+8 \cdot 3^{t} \cdot\left(n_{2}-2\right)\left(n_{2}+1\right)^{t}+2\left(n_{2}-3\right)\left(2 n_{2}+4\right)^{t} \\
& +2\left(n_{2}-2\right)^{2}\left(3 n_{2}+4\right)^{t}+4\left(n_{1}-3\right)\left(4 n_{2}+2\right)^{t} \\
& +\left[2\left(n_{1}-2\right)+4\left(n_{1}-3\right)\left(n_{2}-2\right)\right]\left(4 n_{2}+3\right)^{t} \\
& +4^{t}\left(n_{2}+1\right)^{t}\left[\left(n_{1}-2\right)\left(n_{2}-3\right)+\left(n_{1}-3\right)\left(n_{2}-2\right)^{2}\right]
\end{aligned}
$$

for $n_{1} \geq n_{2} \geq 3$. 
Table 12. The basic information on $G_{r}\left[P_{n_{2}}\right]$.

\begin{tabular}{c|c|c}
$m_{r n_{2}+1, r n_{2}+1}$ & $m_{r n_{2}+1, r n_{2}+2}$ & $m_{r n_{2}+2, r n_{2}+2}$ \\
\hline $2 r n_{1}$ & $2 n_{1}\left[1+r\left(n_{2}-2\right)\right]$ & $n_{1}\left(n_{2}-3\right)+\frac{r n_{1}\left(n_{2}-2\right)^{2}}{2}$
\end{tabular}

Theorem 14. Let $P_{n_{1}}$ and $G_{r}$ be a path and a r-regular of order $n_{1}$ and $n_{2}$, respectively. Then

$$
\operatorname{DTI}\left(P_{n_{1}}\left[G_{r}\right]\right)=r n_{2} \varphi_{r+n_{2}, r+n_{2}}+2 n_{2}^{2} \varphi_{r+n_{2}, 2 n_{2}+r}+\frac{1}{2}\left[r n_{2}\left(n_{1}-2\right)+2\left(n_{1}-3\right) n_{2}^{2}\right] \varphi_{2 n_{2}+r, 2 n_{2}+r}
$$

for $n_{1}>n_{2} \geq 2$.

Proof. By the definition of lexicographic product, we obtain the basic information on $P_{n_{1}}\left[G_{r}\right]$ in the following Table 11.

Table 11. The basic information on $P_{n_{1}}\left[G_{r}\right]$.

\begin{tabular}{c|c|c}
$m_{r+n_{2}, r+n_{2}}$ & $m_{r+n_{2}, 2 n_{2}+r}$ & $m_{2 n_{2}+r, 2 n_{2}+r}$ \\
\hline$r n_{2}$ & $2 n_{2}^{2}$ & $\frac{r n_{2}\left(n_{1}-2\right)}{2}+n_{2}^{2}\left(n_{1}-3\right)$
\end{tabular}

Thus we have

$$
\begin{aligned}
\operatorname{DTI}\left(P_{n_{1}}\left[G_{r}\right]\right) & =\sum_{(i, j) \in K} m_{i, j}(G) \varphi_{i, j} \\
& =r n_{2} \varphi_{r+n_{2}, r+n_{2}}+2 n_{2}^{2} \varphi_{r+n_{2}, 2 n_{2}+r}+\frac{1}{2}\left[r n_{2}\left(n_{1}-2\right)+2\left(n_{1}-3\right) n_{2}^{2}\right] \varphi_{2 n_{2}+r, 2 n_{2}+r} .
\end{aligned}
$$

This completes the proof.

Corollary 14. Let $P_{n_{1}}$ and $G_{r}$ be a path and a r-regular of order $n_{1}$ and $n_{2}$, respectively. Then

$$
\begin{aligned}
& R^{t}\left(P_{n_{1}}\left[G_{r}\right]\right)=r n_{2}\left(r+n_{2}\right)^{2 t}+2 n_{2}^{2}\left(r+n_{2}\right)^{t}\left(2 n_{2}+r\right)^{t}+\left[\frac{r n_{2}\left(n_{1}-2\right)}{2}+\left(n_{1}-3\right) n_{2}^{2}\right]\left(2 n_{2}+r\right)^{2 t}, \\
& Z^{t}\left(P_{n_{1}}\left[G_{r}\right]\right)=2 r n_{2}\left(r+n_{2}\right)^{t-1}+2 n_{2}^{2}\left[\left(r+n_{2}\right)^{t-1}+\left(2 n_{2}+r\right)^{t-1}\right]+\left[r n_{2}\left(n_{1}-2\right)+2\left(n_{1}-3\right) n_{2}^{2}\right]\left(2 n_{2}+r\right)^{t-1}, \\
& \chi^{t}\left(P_{n_{1}}\left[G_{r}\right]\right)=2^{t} r n_{2}\left(r+n_{2}\right)^{t}+2 n_{2}^{2}\left(2 r+3 n_{2}\right)^{t}+2^{t}\left[\frac{r n_{2}\left(n_{1}-2\right)}{2}+\left(n_{1}-3\right) n_{2}^{2}\right]\left(2 n_{2}+r\right)^{t}
\end{aligned}
$$

for $n_{1}>n_{2} \geq 2$.

Theorem 15. Let $G_{r}$ and $P_{n_{2}}$ be a r-regular and a path of order $n_{1}$ and $n_{2}$, respectively. Then

$$
\operatorname{DTI}\left(G_{r}\left[P_{n_{2}}\right]\right)=2 r n_{1} \varphi_{r n_{2}+1, r n_{2}+1}+2 n_{1}\left[1+r\left(n_{2}-2\right)\right] \varphi_{r n_{2}+1, r n_{2}+2}+\frac{1}{2}\left[2 n_{1}\left(n_{2}-3\right)+r n_{1}\left(n_{2}-2\right)^{2}\right] \varphi_{r n_{2}+2, r n_{2}+2}
$$

for $n_{1} \geq n_{2} \geq 3$.

Proof. By the definition of lexicographic product, we obtain the basic information on $G_{r}\left[P_{n_{2}}\right]$ in the following Table 12. Thus we have

$$
\begin{aligned}
\operatorname{DTI}\left(G_{r}\left[P_{n_{2}}\right]\right) & =\sum_{(i, j) \in K} m_{i, j}(G) \varphi_{i, j} \\
& =2 r n_{1} \varphi_{r n_{2}+1, r n_{2}+1}+2 n_{1}\left[1+r\left(n_{2}-2\right)\right] \varphi_{r n_{2}+1, r n_{2}+2}+\frac{1}{2}\left[2 n_{1}\left(n_{2}-3\right)+r n_{1}\left(n_{2}-2\right)^{2}\right] \varphi_{r n_{2}+2, r n_{2}+2}
\end{aligned}
$$

This completes the proof. 
Corollary 15. Let $G_{r}$ and $P_{n_{2}}$ be a r-regular and a path of order $n_{1}$ and $n_{2}$, respectively. Then

$$
\begin{aligned}
R^{t}\left(G_{r}\left[P_{n_{2}}\right]\right)= & 2 r n_{1}\left(r n_{2}+1\right)^{2 t}+2 n_{1}\left[1+r\left(n_{2}-2\right)\right]\left[\left(r n_{2}+1\right)\left(r n_{2}+2\right)\right]^{t} \\
& +\left[n_{1}\left(n_{2}-3\right)+\frac{r n_{1}\left(n_{2}-2\right)^{2}}{2}\right]\left(r n_{2}+2\right)^{2 t}, \\
Z^{t}\left(G_{r}\left[P_{n_{2}}\right]\right)= & 4 r n_{1}\left(r n_{2}+1\right)^{t-1}+2 n_{1}\left[1+r\left(n_{2}-2\right)\right]\left[\left(r n_{2}+1\right)^{t-1}+\left(r n_{2}+2\right)^{t-1}\right] \\
& +\left[2 n_{1}\left(n_{2}-3\right)+r n_{1}\left(n_{2}-2\right)^{2}\right]\left(r n_{2}+2\right)^{t-1}, \\
\chi^{t}\left(G_{r}\left[P_{n_{2}}\right]\right)= & 2^{t+1} r n_{1}\left(r n_{2}+1\right)^{t}+2 n_{1}\left[1+r\left(n_{2}-2\right)\right]\left(2 r n_{2}+3\right)^{t} \\
& +2^{t}\left[n_{1}\left(n_{2}-3\right)+\frac{r n_{1}\left(n_{2}-2\right)^{2}}{2}\right]\left(r n_{2}+2\right)^{t}
\end{aligned}
$$

for $n_{1} \geq n_{2} \geq 3$.

Theorem 16. Let $G_{1}$ and $G_{2}$ be a $r_{1}$-regular graph and a $r_{2}$-regular graph with order $n_{1}$ and $n_{2}$, respectively. Then

$$
\operatorname{DTI}\left(G_{1}\left[G_{2}\right]\right)=\frac{1}{2} n_{1} n_{2}\left(r_{2}+r_{1} n_{2}\right) \varphi_{r_{1} n_{2}+r_{2}, r_{1} n_{2}+r_{2}}
$$

for $n_{1} \geq n_{2} \geq 2$.

Proof. By the definition of lexicographic product, we have $G_{1}\left[G_{2}\right]$ is a $\left(r_{1} n_{2}+r_{2}\right)$-regular graph. Thus

$$
\operatorname{DTI}\left(G_{1}\left[G_{2}\right]\right)=\sum_{(i, j) \in K} m_{i, j}(G) \varphi_{i, j}=\frac{1}{2} n_{1} n_{2}\left(r_{2}+r_{1} n_{2}\right) \varphi_{r_{1} n_{2}+r_{2}, r_{1} n_{2}+r_{2}} .
$$

This completes the proof.

Corollary 16. Let $G_{1}$ and $G_{2}$ be a $r_{1}$-regular graph and a $r_{2}$-regular graph with order $n_{1}$ and $n_{2}$, respectively. Then

$$
\begin{aligned}
& R^{t}\left(G_{1}\left[G_{2}\right]\right)=\frac{n_{1} n_{2}\left(r_{2}+r_{1} n_{2}\right)^{2 t+1}}{2}, \\
& Z^{t}\left(G_{1}\left[G_{2}\right]\right)=n_{1} n_{2}\left(r_{2}+r_{1} n_{2}\right)^{t}, \\
& \chi^{t}\left(G_{1}\left[G_{2}\right]\right)=2^{t-1} n_{1} n_{2}\left(r_{2}+r_{1} n_{2}\right)^{t+1}
\end{aligned}
$$

for $n_{1} \geq n_{2} \geq 2$.

\section{Conclusion}

In this paper, we give a unified approach to solve the computational problems of degree-based topological indices of standard product graphs for the path, star and regular graphs. It is imaginable to use other graph operations to calculate degree-based topological indices uniformly in the future.

Acknowledgments: This work was supported by the Qinghai science and technology plan project (No. 2021-ZJ-703) and the National Natural Science Foundation of China (No. 11771443).

Author Contributions: All authors contributed equally to the writing of this paper. All authors read and approved the final manuscript.

Conflicts of Interest: "The authors declare no conflict of interest."

\section{References}

[1] Todeschini, R., \& Consonni, V. (2009). Molecular Descriptors for Chemoinformatics. Wiley-VCH, Weinheim.

[2] Bollobás, B., \& Erdős, P. (1998). Graphs of extremal weights. Ars Combinatoria, 50, 225-233.

[3] Randić, M. (1975). On characterization of molecular branching. Journal of the American Chemical Society, 97, 6609-6615.

[4] Gutman, I., Furtula, B., \& Elphick, C. (2014). Some new/old vertex-degree-based topological indices. MATCH Communications in Mathematical and in Computer Chemistry, 72, 617-632. 
[5] Gutman, I., \& Trinajstić, N. (1972). Graph theory and molecular orbitals, Total $\pi$ electron energy of alternant hydrocarbons. Chemical Physics Letters, 17, 535-538.

[6] Li, X., \& Zheng, J. (2005). A unifled approach to the extremal trees for difierent indices. MATCH Communications in Mathematical and in Computer Chemistry, 54, 195-208.

[7] Furtula, B., \& Gutman, I. (2015). A forgotten topological index. Journal of Mathematical Chemistry, 53, 1184-1190.

[8] Zhou, B., \& Trinajstić, N. (2010). On general sum-connectivity index. Journal of Mathematical Chemistry, 47, $210-218$.

[9] Fajtlovicz, S. (1987). On conjectures on Graffiti-II. Congr numer, 60, 187-197.

[10] Zhou, B., \& Trinajstić, N. (2009). On a novel connectivity index. Journal of Mathematical Chemistry, 46, $1252-1270$.

[11] Das, K. Ch., Gutman, I., Milovanović, I., Milovanović, E., \& Furtula, B. (2018). Degree-based energies of graphs. Linear Algebra and Its Applications, 554, 185-204.

[12] Imrich, W., \& Klavžar, S. (2000). Product Graphs. Structure and Recognition, Wiley-Interscience. New York.

[13] Feder, T. (1995). Stable networks and product graphs. Memoirs of the American Mathematical Society, 116, 555.

[14] Lammprey, R. H., \& Barnes, B. H. (1974). Products of graphs and applications. Modeling and Simulation, 5, 1119-1123.

[15] Ghozati, S. A. (1999). A finite automata approach to modeling the cross product of interconnection networks. Mathematical and Computer Modelling, 30, 185-200.

(C) 2021 by the authors; licensee PSRP, Lahore, Pakistan. This article is an open access article distributed under the terms and conditions of the Creative Commons Attribution (CC-BY) license (http://creativecommons.org/licenses/by/4.0/). 